\title{
Unhealthy consumerism: The challenge of trading off price and quality in health care
}

\author{
KATE BARASZ* \\ IESE Business School, Barcelona, Spain \\ PETER A. UBEL \\ Fuqua School of Business, Sanford School of Public Policy, Duke-Margolis Center for Health Policy and School \\ of Medicine, Duke University, Durham, NC, USA
}

\begin{abstract}
Over the last decade, health care in many parts of the world has shifted toward a more patient-centric, consumeristic model, marked by an emphasis on choice and a proliferation of typical consumer-facing information (e.g. price and quality data). However, while the 'patients as consumers' perspective is an apt one, there are crucial differences between health care and typical consumer domains that warrant special consideration by policy-makers and researchers alike. This article discusses some of these differences and explores the challenges that consumers (i.e. patients) face when making trade-offs between price and quality.
\end{abstract}

Submitted 21 December 2016; accepted 26 January 2017

Suppose you are in the market for a new car. Before spending \$30,000, you likely weigh your options carefully and - as you would in most typical consumer domains - consider the trade-offs between price and quality: what can you get for what you can pay? To answer this, an ample amount of price and quality information awaits you: safety ratings, expert and consumer reviews, value retention estimates and reliability reports. These data, coupled with well-established quality metrics (e.g. fuel economy, crash test results and average cost of maintenance), make the decision process relatively straightforward: you can maximize the dimension(s) you deem most important and choose a car about whose price you feel comfortable and quality you feel confident.

But now suppose you are spending $\$ 30,000$ on a new knee. How might your decision process change? Over the past several decades, health care delivery in

\footnotetext{
*Correspondence to: IESE Business School, Barcelona, Spain. Email: kbarasz@iese.edu
} 
many parts of the world has evolved from treating patients as passive health care recipients to seeing them as empowered, decision-making health care consumers. In many ways, this 'patients as consumers' perspective is an apt one: choices that once fell entirely within the physician's jurisdiction are being pushed to patients, and today's health care consumers increasingly face decisions about everything from who should provide their care (e.g. where and by whom to be seen) to what care they should receive (e.g. which procedures, tests, screenings and medications to ask for). Consequently, patients seeking a knee replacement may expect to have the final say around if and when to have the surgery, exactly what type of replacement they want, and which surgeon and surgical center they will use.

To make these decisions, patients may use price and quality information as the basis for their choice - just as they would for other consumer decisions. How much will the knee replacement cost and does this vary between providers? Which providers have the best outcomes or the lowest risks of complications? What is the optimal trade-off between out-of-pocket expense and better health results? As the patient's role as decision-maker has expanded, so too has the amount of information available to help them make these choices. Like car buyers, today's health care consumers can access an assortment of price and quality data: patient ratings and reviews (e.g. sites like Healthgrades in the USA or iWantGreatCare in the UK), hospital quality evaluations (from government agencies like the Centers for Medicare and Medicaid Services in the USA or the National Health Service in the UK) and price information (e.g. sites like Healthcare Bluebook in the USA). With so many seeming parallels, this again begs the question: to what extent do patients shopping for knee replacements behave like consumers shopping for cars? Further, if patients are becoming more like typical consumers - and we already know how typical consumers behave - should health care policies and platforms be designed to emulate usual consumption contexts?

While it is certainly reasonable to draw some comparisons between the health care domain and other consumer contexts - knee replacement buyers surely exhibit some of the same discerning behaviors as car buyers - there are important limits to this analogy. Regardless of how 'consumeristic' the health care pendulum swings, there remain fundamental dissimilarities between health care settings and typical consumer contexts that make health care decisions distinct (e.g. differences in the stakes and context of the choice). Further, price and quality information in the health care domain is not as straightforward - both in terms of what is communicated and how it is interpreted and applied - as it is in other consumer domains. As such, there are reasons to believe that health care consumers may not behave exactly like decision-makers in typical consumer contexts. To regard health 
care decisions as more or less analogous to typical consumer decisions - both in how policies and programs are designed as well as what consumer behavior is expected - would be to miss some important nuances that may have unintended consequences in choice.

In this article, we discuss several crucial ways in which the health care context differs from other typical consumer contexts, exploring some of the potential challenges and pitfalls in how consumers use information to make health care choices. Specifically, we focus our discussion on the use of price and quality information in health care and its impact on decisions around providers and care. As the consumer's role in health care decision-making grows and access to decision-relevant information expands, understanding this choice process is a critical first step in designing thoughtful programs and policies. Whether the objective is to empower patients to make choices that align with their personal values, to determine if and how to provide quality and price information to consumers, or to encourage specific 'desirable' decisions (e.g. steering consumers toward more cost-effective providers and procedures), there are dynamics - both known and yet to be researched - that should be considered.

\section{The health care consumer context}

Though the health care setting increasingly resembles other consumer contexts with its emphasis on choice and 'shopping around', it remains unique in many respects. Unlike typical consumer domains, health care decisions often have distinct characteristics (e.g. time sensitive, high stakes, infrequent and emotionally wrought). Indeed, even if the actual 'buying process' for a new knee perfectly approximated the buying process for a new car, there would still be additional considerations that may meaningfully change the consumer's role in the decision process.

For example, consider initiating the search process either for a new car or a new knee. An important first step is knowing what you are looking for: which dimensions should you prioritize and how should you think about price and quality trade-offs? In typical consumer contexts, gathering this knowledge is relatively straightforward, informed by your own previous experience (e.g. maybe you had reliability issues with your last vehicle and want to ensure your new car has fewer mechanical troubles), sampling the product directly (e.g. you can test drive the cars you ultimately want to buy) or seeking recommendations from fellow consumers (e.g. reading reviews of the make and model you are considering). Knee replacement patients, on the other hand, are often first-time consumers, lacking expertise and prior experience; you cannot test drive a new knee. Further, even if you ask other knee replacement 
patients about their own experiences, results vary between patients; knee replacements are not standardized goods rolling off an assembly line. Toyota sells hundreds of thousands of Camrys each year, so consumers have a wealth of information on the reliability of those vehicles. By contrast, an orthopedic surgeon might perform 50 or 100 knee replacement procedures in a year, with the result that data on surgeons' success rates will be much less precise. For these reasons, health care consumers face their decisions with less established, more uncertain a priori preferences and values; as such, they may be less willing or able to engage in an extensive information search, less confident in their choice and more sensitive to outside sources of information or influence.

Further, consider the context in which health care versus typical consumer decisions are made. Car buyers generally have the flexibility to shop around and consider options over time. They face decisions that are high stakes in a financial sense, and there may be uncertainty around how they will ultimately like their new vehicles, but the decisions are not life or death. Some patient decisions, on the other hand, may very well be; many medical decisions are time sensitive - patients may not have months to shop around for health care providers, but only days or weeks or potentially no time at all. And medical decisions are often high-stakes choices, not only in the financial sense, but also because of risks to health and well-being. In these ways, health care consumers almost certainly approach decisions from a different starting point than typical consumers. For instance, they may view trade-offs in very different ways (e.g. whereas a car consumer may not be willing to pay twice as much for a vehicle that is only slightly better, a knee replacement patient may see marginal improvements as worthwhile at any cost) and feel more uncertainty and anxiety around their choice.

Finally, consider the interpersonal dynamics at play in making medical decisions. A car buyer can reflect on her priorities and conduct due diligence about her purchase before entering the showroom; car dealers can certainly affect the choice, but the buyer is typically aware of the salesperson's ulterior motives and incentives and may in turn be less susceptible to their influence (Campbell \& Kirmani, 2000). A patient, on the other hand, approaches the decision from a different place in terms of expertise, power and awareness of possible ulterior motives or conflicts of interest. Because health care consumers may not know what exactly they are looking for and may feel a great deal of time pressure, anxiety and uncertainty around the choice, they are far more susceptible to the advice of their physicians (Gurmankin et al., 2002) and largely blind to potential conflicts of interest (Cain et al., 2005). In fact, treatment decisions often reflect the recommendations of physicians more than they reflect the values of the patients themselves (Scherr et al., 2017). Therefore, 
regardless of how much diligence consumers do beforehand, values, objectives and decisions may change entirely once inside the exam room.

With these underlying contextual differences in mind, we next explore the specific ways in which consumers' interpretations and use of price and quality information are likely to differ in health care settings versus other typical consumer domains.

\section{The mixed messages of price}

Some health care systems are more consumer-oriented than others. The British health care system, for example, is not particularly consumer oriented; patients have little incentive to explore the price of health care because they do not pay out of pocket for their care. By contrast, many patients in Singapore and the United States are expected to pay a significant portion of the price out of pocket and as such are more motivated to learn about the prices of their medical care. Accordingly, health care procedures - similar to cars or household appliances - are increasingly acquiring price tags that consumers can scrutinize.

Making this price information salient before health care services are rendered - or implementing health care price transparency - has become an increasingly popular policy proposition in the United States, both as a way to make patients better informed and to drive down health care costs more generally (Sinaiko \& Rosenthal, 2011). The idea is that when consumers are searching for information about providers and care, they can use price information - which highlights the literal cost of certain trade-offs - to make better-informed decisions. Health care price transparency enables patients/consumers to ask questions, such as: is it really worthwhile to spend four times more money on a premium drug that is only marginally more effective than the next best option?

The benefits of presenting price information may be numerous. First, price can be a relatively straightforward and easily interpretable metric. People have ample experience with price information, which should make trade-off decisions easier; in everything from buying a new car ("Would I be willing to pay $\$ 3,000$ more for Safety Feature X?”) to buying a pint of ice cream ("Should I spend \$2 more for the branded tub of cookies and cream?"), price-quality trade-offs are both familiar and concrete. The same dynamic may hold true in health care domains. For example, imagine a choice between Physician A, who charges $\$ 37,000$ and has an $85 \%$ success rate, or Physician B, who charges $\$ 30,000$ and has an $80 \%$ success rate. Patients have some familiarity making these types of trade-offs ("Would I be willing 
to pay $\$ 7,000$ more for a success rate that is five points higher?”), which may help ease health care consumer decisions.

Further, if policy-makers have the objective of driving down health care costs, price information could be an important lever to use. In the US health care system, there is incredible variation between prices for identical procedures and tests (Cooper et al., 2015) with little to no accompanying evidence that higher prices yield better quality or outcomes (e.g. Hussey et al., 2013; Huntington et al., 2015). For example, one study in the United States showed hip replacement prices ranging from $\$ 10,000$ to over $\$ 100,000$ for the procedure (Rosenthal et al., 2013). Publishing prices has therefore been put forth as one way to drive down variability and allow consumers to make more cost-conscious choices. For instance, Healthcare Bluebook, a private US company that aggregates health care price information, operates with the mission of creating a fairer marketplace by exposing unnecessary price differences and allowing consumers to shop around; a patient who needs a chest X-ray can look up the 'going rate' in her area to ensure she is not being overcharged.

But despite its similarities to other consumer contexts, health care prices may not prompt the same response as prices in typical consumer domains; health care has unique structural factors that may change the ways patients use and interpret price information (Sinaiko \& Rosenthal, 2011). First, there is the issue of which price health care providers should publish, itself a thorny and complicated consideration (Cutler \& Dafny, 2011). Consumers can readily find the price of a new laptop or estimate the price of a nice dinner out for two. Cars have sticker prices that - even if they are ultimately negotiated serve as an identical starting point for everyone. However, because of the structure of health care pricing - at least in the United States - hospitals and physicians may charge completely different rates as a function of their negotiated contracts with insurers. A surgery may have a 'list price' of $\$ 30,000$, but one patient's insurance may actually pay $\$ 5,000$, another patient's insurance may pay $\$ 20,000$ and an uninsured patient may be responsible for the full $\$ 30,000$. Therefore, unlike in other consumer domains, health care has the added complexity of establishing what the actual price is.

Beyond that, prices are further obscured by insurance, another structural dynamic unique to the health care context and not a consideration in most typical consumer domains. Assume, for simplicity, that all health care procedures or tests had one transaction price per provider: Physician A charged $\$ 37,000$ and Physician B charged $\$ 30,000$ to any and all health care consumers (i.e. they did not negotiate separate prices by insurance plan). Even in this unrealistically simple scenario, there would still remain the issue of what price patients themselves have to pay. For instance, one patient may have a 
copay insurance plan that allows them to pay a flat rate of $\$ 100$ regardless of whether they chose Physician A or B, while another patient may be responsible for paying the whole bill in full. It is easy to imagine how these differences in out-of-pocket expenses may also change how patients factor prices into their ultimate decisions: for instance, a health care consumer with a flat-rate copay may choose the more expensive Physician A, while the full-price patient may be extremely sensitive to price differences and select the lowerpriced Provider B. This price structure may result in idiosyncratic decisions, making the role of price difficult to generalize across health care consumers.

A final structural consideration is how and where health care prices are communicated. In typical consumer contexts, people may ascertain price information in a variety of ways - whether it is published directly on the good or determined by inquiring with a salesperson - but regardless of how it is obtained, discussing price is an almost universally acceptable and established part of any consumer transaction. But not so with prices in the health care context. Prices have long been relegated to an afterthought in medical decision-making and, as such, many physicians do not take the opportunity to discuss costs with their patients (Ubel et al., 2016). Further, unlike a new laptop or dishwasher, whose prices can be researched before the transaction decision is ever made, health care prices are often most relevant at awkward and inopportune moments; when discussing a cancer treatment that may save your child's life, inquiring about the price of the drug may seem trivial or, worse, taboo. Thus, even if they are available, how and when health care prices are communicated may change how patients factor them in relative to other consumer contexts.

In addition to the structural factors unique to the health care context, prices can also change people's decisions by sending a signal about quality. This fact is not only relevant for health care; a substantial body of behavioral research has demonstrated that price actively shapes people's perceptions of quality, such that higher prices often signal higher quality or, conversely, that lower prices may signal lower quality (Rao \& Monroe, 1989; Shiv et al., 2005; Waber et al., 2008). For example, researchers found that a $\$ 90$ bottle of wine reportedly tasted better - and even elicited different neural responses than the same exact bottle priced at $\$ 10$ (Plassmann et al., 2008). The same may be true in health care, as well. For instance, efforts are underway to decrease the price of in vitro fertilization (IVF) procedures by eliminating unnecessary expense while only minimally sacrificing odds of success. But while the move could make IVF treatments more affordable to infertile couples, experts worry that "dispelling the notion that low cost means low quality will be a challenge" (The Economist, 2016) - and hence the paradox: people would almost always prefer the same good at a more affordable 
price, but that more affordable price may signal that it is not, indeed, the same good.

Although not unique to health care, this 'higher price means higher quality' inference may be particularly consequential in medical decisions. Policymakers want to use price transparency to lower health care costs via creating more savvy, cost-conscious patients who shop around for better-priced care (Sinaiko \& Rosenthal, 2011; Emanuel et al., 2012). However, if price inadvertently communicates something about quality, it is possible that the exact opposite effect could occur. If patients value quality (as they almost certainly do), they may be motivated to choose higher-priced - not lower-priced - providers (Hibbard et al., 2012). For a policy-maker tasked with driving down health care costs, this unforeseen dynamic may have truly adverse consequences, which epitomizes one of the key challenges of presenting health care price information: how, exactly, will consumers interpret it? And will the interpretation encourage desirable decisions or have unforeseen side effects?

As health care becomes more consumer-oriented, price will undoubtedly figure in as a data point that consumers both demand and incorporate into their medical decisions. However, there are structural factors unique to the health care setting (i.e. varying provider pricing for identical goods or procedures, varying out-of-pocket expenses for consumers as a function of their insurance plans, and the difficulty of communicating and incorporating price into health care decisions) and a risk of inadvertently steering patients toward more expensive providers through price transparency. Therefore, policy-makers and researchers must be thoughtful of the differences between how health care consumers use price relative to other consumer contexts.

\section{The complexity of quality}

If displaying health care prices inadvertently (and incorrectly) insinuates something about quality, might publishing actual quality data mitigate this concern? For example, think about a choice between two orthopedic surgeons performing knee replacements and assume you only knew the following: Physician A charges $\$ 37,000$ and Physician B charges $\$ 30,000$. Were you to use price as an indicator of quality, you may be tempted to choose the former, assuming that the higher price was justified by better outcomes. But imagine how your choice might change if prices were paired with quality data and you learned that lower-priced Physician B's quality was equal to higher-priced Physician A. In an ideal world, this would induce the kind of savvy, cost-conscious health care decisions that policy-makers hope for: why not pay $\$ 7,000$ less for something that is just as good? 
While this solution seems straightforward, providing health care quality information is a complex endeavor. For example, what metrics would fairly capture Physician A and B's quality? Just as there is ambiguity in determining which health care price to provide, there is also ambiguity in determining which measures of health care quality to report. Should quality metrics reflect objective health outcomes or subjective patient satisfaction scores, complications and risks or effectiveness and successes? All of these considerations again differentiate the health care context from typical consumer settings. When buying a car, there are established and readily available measures that consumers can use to gauge quality (e.g. safety ratings, fuel economy, maintenance costs, etc.), but the complexity of health care quality information is daunting. The United States' Centers for Medicare and Medicaid Services (CMS), for example, reports an overall 'quality score' for each hospital based on 64 different quality measures that fall within seven broader categories (e.g. mortality, safety of care and effectiveness of care; Hospital Compare, 2016). From the health care provider's standpoint, what is a fair way to measure quality?

Moreover, from the health care consumer standpoint, which measure of quality should they care most about? The contextual factors discussed above - patients' uncertainty around their own preferences and values and the high stakes and emotionality of the decision - make evaluating quality a unique undertaking in the health care space. To compare, think about evaluating the quality of a car; although there are many available metrics, consumers may intuitively have a sense for which metrics they care most about. A consumer with small children may care most about the safety ratings, while a consumer on a tight budget may care most about minimizing maintenance costs; consumers can acquire enough expertise to more or less know their priorities. However, lacking health care expertise and previous experience, patients may not have these same intuitions about which attribute matters most to their experience and their outcomes; surely a good bedside manner will make the process more pleasant, but does that have anything to do with how their newly replaced knee will function post-operatively?

Further complicating this is the fact that even if health care consumers purport to know which quality measures they care about, this may not always align with how they choose. A McKinsey consumer survey asked patients about their health care experiences and which dimensions of quality mattered most to them: over $90 \%$ of respondents said they cared most about the health outcomes achieved, but further analysis revealed that bedside manner (e.g. empathy and support of health care professionals) was a much stronger predictor of patient satisfaction (Cordina et al., 2015). This disconnect is not specific to health care - psychologists have long acknowledged the gap between what people say and how they behave (Nisbett $\&$ Wilson, 
1977) - but it presents a unique complication when trying to narrow down which quality metrics are most helpful in aiding health care choices.

Another consideration is the potential influence policy-makers and health care providers have on shaping which dimensions of quality patients prioritize: do health care consumers know their priorities before accessing quality measures, or do the quality measures ex post facto determine their priorities? Behavioral research makes a strong case for the latter. A broad literature has shown the extent to which consumers' preferences are influenced by contextual factors and cues (e.g. Slovic, 1995; Payne et al., 1999). A patient may, for example, initially believe she cares most about minimizing negative surgical outcomes, but end up selecting the doctor who has the best bedside manner. A variety of inputs shape what consumers ultimately choose, and preferences can be significantly shaped by seemingly trivial contextual factors, such as the order in which metrics are displayed (Levav et al., 2010) or whether they are displayed individually or alongside others (Hsee et al., 1999). These contextual factors are especially influential when people's previous experience or expertise is low (Fischhoff et al., 1980) - as is so often the case in health care. Again, this is not necessarily unique to the health care setting, but when you have decision-makers who lack expertise and a clear a priori notion of their values and preferences, the effect may be more pronounced. Health care consumers are likely to be influenced - knowingly or not - by the metrics themselves, in which case policy-makers have an ethical obligation to thoughtfully curate the quality measures that are displayed.

For example, consider the UK's National Health Service (NHS), which offers the 'NHS Choices' portal. Here, consumers can search by procedure and location to shop around for health care providers, with sortable quality information provided alongside. For knee replacements, the site displays three key objective quality metrics: time between referral and treatment; number of knee replacements performed in the previous 12 months (by replacement type); and length of hospital stay (NHS Choices, 2016). These three metrics may encapsulate all - or absolutely none - of the quality metrics consumers initially thought they cared about, but once they have encountered this information, consumers will likely incorporate it into their decision regardless (Kleinmuntz \& Schkade, 1993; Payne et al., 1999). Maybe they think the time between referral and treatment is something they should care about, even if they did not previously. Consequently, consumers who cared nothing about the lag time between referral and surgery may suddenly find themselves striking a balance between this metric and the two others, leaving the decision with different revealed preferences than they started with. As a result, whatever policy-makers or health care professionals decide to display - which may be evidence based and purposeful, or merely 
an artifact of what information was available - will likely become influential in how consumers make their decisions.

Furthermore, the process for making decisions and trade-offs may differ depending on the format in which the quality information is presented. Compare the NHS's approach to the United States' CMS, which also aggregates hospital and physician quality information (Hospital Compare, 2016). Rather than the NHS's numeric format, the CMS's Hospital Compare summarizes quality information in star ratings; hospitals can earn between one and five stars based on 64 aggregated measures of quality. Though the NHS and CMS both deliver objective quality metrics to their consumers, it is easy to imagine how - even holding constant a consumer and her preferences - variations in these two formats could lead to different choices or trade-offs. A patient can readily envision the experience of (and perhaps articulate his/her preferences for) a three-night hospital stay versus a five-night hospital stay, or imagine the implications of a physician having performed 10 knee replacements versus 100, but the trade-off between five stars and four stars is much less concrete. Notionally, consumers understand there are underlying quality differences between four stars and five, but it is more difficult to comprehend what, exactly, is gained or lost. On the other hand, there may be value in summarizing and simplifying via star ratings to reduce the burden on consumers. Either way, consumers' trade-offs between different quality dimensions is likely to vary as a function of the format in which it is displayed.

In addition to thinking about trade-offs among more objective measures of quality, there are also subjective quality measures to consider, such as selfreported patient satisfaction levels. The CMS incorporates patient experience into their overall quality metrics, measuring factors like 'Patients who reported that their doctors communicated well' and 'Patients who reported that they received help as soon as they wanted' (Hospital Compare, 2016). Likewise, the NHS allows consumers to assign star ratings based on factors like cleanliness, staff cooperation, dignity and respect and involvement in decisions (NHS Choices, 2016). Private companies (e.g. the publication U.S. News \& World Report, the US website Healthgrades, and the UK website iWantGreatCare) have also joined the game, collecting and publishing reviews of hospitals and physicians through their own platforms.

This proliferation of health care provider reviews raises further questions about how consumers make trade-offs in quality. For instance, consumers may prefer Physician A's $85 \%$ surgical success rate to Physician B's $80 \%$ surgical success rate, but might that flip if they discover that Physician A has a three-star patient rating and Physician B has a five-star rating? In today's connected world, many consumption decisions (e.g. for restaurants, hotels, gadgets and books) maximize such ratings, but it is less clear that these same 
ratings will result in better medical decisions. In selecting a restaurant, people are consuming and presumably trying to maximize the experience, which makes reading about others' experiences well aligned with the decision goal; a three-star rating earned by delivering poor service but good food can still sour the restaurant experience, which may rightly deter consumers from dining there. However, when making decisions about health care, the experience is only one part of the equation; a three-star rating earned by a brusque bedside manner but solid medical care does not sour the medical experience in the same way, particularly if maximizing health is the superseding objective.

Moreover, health care provider reviews and ratings are by their very nature subjective, but made more so by underlying and largely unobservable factors. For instance, what was the condition or illness for which the reviewer (i.e. patient) was being treated? Patients facing the most challenging medical conditions are the hardest to help, will statistically have worse outcomes (Chassin et al., 1996) and perhaps accordingly have the lowest satisfaction rates - all of which skew the ratings and quality outcomes of those physicians and hospitals who take on the sickest cases. Understanding the many ways that consumers weigh and factor in subjective versus objective quality ratings is another rich area for investigation.

Clearly, the complexities of incorporating quality information into consumers' health care decisions are many. However, if the goal is to empower consumers, incentivize quality or simply understand the consequences - intended and otherwise - of publishing such information, an attempt should be made to understand how consumers process and weigh quality information, specifically considering: (1) how consumers define and prioritize dimensions of quality; (2) how they make trade-offs between different quality dimensions; (3) and how these trade-offs may change as a function of format (e.g. numeric versus star ratings) and content (e.g. quantitative metrics versus subjective reviews).

\section{Discussion and policy implications}

We have laid out a series of decision-making challenges that undermine the ability of health care consumers (also known as patients) to make trade-offs between price and quality. Even as the health care domain becomes more consumeristic, and price and quality information become increasingly central to patient decision-making, there remain fundamental differences between how patients and typical consumers will make trade-offs and decisions. The scope of these challenges raises an important policy question: should we adopt policies that minimize the role of patients as consumers?

Our short answer is - yes and no. 
Yes, we should dial back our expectations of health care consumerism in contexts where it is impossible for patients to carefully scrutinize price and quality. A person with crushing substernal chest pain should not be expected to compare the price of local angioplasty centers before calling 911 and telling the ambulance where to go. If we are asking patients to pay out of pocket for emergency services, we might be doing so for economic reasons to share the burden of those health care costs - but we should not do so because we think it will bring consumer savvy to those health care choices.

Nor should we push for policies that increase people's out-of-pocket expenses without simultaneously making it easier for them to get comprehensible information about the cost and quality of health care providers. Without a better understanding of how patients interpret and use price information - and without a straightforward, unbiased way of presenting accompanying quality data - it is premature to force patients into typical consumer roles and expect typical consumer behaviors to prevail.

Of course, while we have argued that price and quality information will not, on their own, necessarily lead to optimal health care choices, there are nevertheless reasons to support policies that make patients more like consumers by asking them to pay a meaningful portion of their health care expenses out of pocket. Without such economic incentives, people demand (or passively accept) overly expensive and potentially unnecessary medical care. Moreover, even in systems like those of the UK, where people do not usually pay out of pocket for their medical care, good medical care still relies upon patients taking on a role as proactive health care consumers. Even if a knee replacement is free, it might not be in a person's best interest to undergo the surgery; the patient needs to get involved in her health care choice well enough to understand whether the procedure will make her life better or worse. Similarly, she needs to decide which surgeon to go to for the procedure; one surgeon might have a better reputation and a higher quality rating but a much longer wait time, and that trade-off should ultimately be up to the patient.

We point out some of the challenges of health care consumerism not to argue for policies that minimize the role of patients as consumers. Health care consumerism is unavoidable. Instead, we urge policy-makers to recognize the limitations of health care consumerism - the many ways in which patients are not going to behave with the same kind of consumer savvy that they exert in other parts of the consumer economy - and enact policies accordingly.

\section{References}

“An Arm and a Leg for a Fertilised Egg; In Vitro Fertilisation" (2016) The Economist. 
Cain, D.M., G. Loewenstein, D.A. Moore (2005), 'The Dirt on Coming Clean: Perverse Effects of Disclosing Conflicts of Interest', The Journal of Legal Studies, 34: 1-25.

Campbell, M.C., A. Kirmani (2000), 'Consumers' Use of Persuasion Knowledge: The Effects of Accessibility and Cognitive Capacity on Perceptions of an Influence Agent,' Journal of Consumer Research, 27: 69-83.

Chassin, M.R., E.L. Hannan, B.A. Debuono (1996), 'Benefits and Hazards of Reporting Medical Outcomes Publicly', New England Journal of Medicine, 334: 394-398.

Cooper, Z., S.V. Craig, M.S. Gaynor, J. Reenan (2015), 'The Price Ain't Right? Hospital Prices and Health Spending on the Privately Insured', National Bureau of Economic Research, http:// www.nber.org/papers/w21815. (Accessed: 7 Dec 2016).

Cordina, J., R. Kumar, C. Moss (2015), 'Debunking Common Myths About Healthcare Consumerism', McKinsey \& Company.

Cutler, D., L. Dafny (2011), 'Designing Transparency Systems for Medical Care Prices', New England Journal of Medicine, 364: 894-895.

Emanuel, E., N. Tanden, S. Altman, S. Armstrong, D. Berwick, F. de Brantes, M. Calsyn, M. Chernew, J. Colmers, D. Cutler, T. Daschle, P. Egerman, B. Kocher, A. Milstein, E. Oshima Lee, J.D. Podesta, U. Reinhardt, M. Rosenthal, J. Scharfstein, S. Shortell, A. Stern, P.R. Orszag, T. Spiro (2012), 'A Systematic Approach to Containing Healthcare Spending', New England Journal of Medicine, 367: 949-954.

Fischhoff, B., P. Slovic, S. Lichtenstein (1980), 'Knowing What You Want: Measuring Labile Values', in: Wallsten, T.S. (Ed.), Cognitive Processes in Choice and Decision Behavior, Lawrence Erlbaum, Hillsdale, NJ.

Gurmankin, A.D., J. Baron, J.C. Hershey, P.A. Ubel (2002), 'The Role of Physicians' Recommendations in Medical Treatment Decisions', Medical Decision Making, 22: 262-271.

Hibbard, J.H., J. Greene, S. Sofaer, K. Firminger, J. Hirsh (2012), 'An Experiment Shows That A Well-Designed Report On Costs And Quality Can Help Consumers Choose High-Value Health Care', Health Affairs, 31: 560-568.

Hospital Compare (2016) Centers for Medicare and Medicaid Services, https://www.medicare.gov/ hospitalcompare/search.html (Accessed: 10 Sept 2016).

Hsee, C.K., G. Loewenstein, S. Blount, M.H. Bazerman (1999), 'Preference Reversals Between Joint and Separate Evaluations of Options: A Review And Theoretical Analysis', Psychological Bulletin, 125: 576-590.

Huntington, C.R., T.C. Cox, L.J. Blair, T. Prasad, A.E. Lincourt, B.T. Heniford, V.A. Augenstein (2015), 'Nationwide variation in outcomes and cost of laparoscopic procedures', Surgical Endoscopy, 30: 934-946.

Hussey, P.S., S. Wertheimer, A. Mehrotra (2013), 'The Association Between Health Care Quality and Cost', Annals of Internal Medicine, 158: 27-34.

Kleinmuntz, D.N., D.A. Schkade (1993), 'Information Displays And Decision Processes', Psychological Science, 4: 221-227.

Levav, J., M. Heitmann, A. Herrmann, S. Iyengar (2010), 'Order in Product Customization Decisions: Evidence from Field Experiments’, Journal of Political Economy, 118: 274-299.

NHS Choices (2016) National Health Service, http://www.nhs.uk/pages/home.aspx (Accessed 10 Sept 2016).

Nisbett, R.E., T.D. Wilson (1977), 'Telling more than we can know: Verbal reports on mental processes', Psychological Review, 84: 231-259.

Payne, J.W., J.R. Bettman, D.A. Schkade (1999), 'Measuring Constructed Preferences: Towards Building a Code', Journal of Risk and Uncertainty, 19: 243-270.

Plassmann, H., J. O’Doherty, B. Shiv, A. Rangel (2008), 'Marketing Actions Can Modulate Neural Representations of Experienced Pleasantness', Proceedings of the National Academy of Sciences, 105: 1050-1054. 
Rao, A.R., K.B. Monroe (1989), 'The Effect of Price, Brand Name, and Store Name on Buyers' Perceptions of Product Quality: An Integrative Review', Journal of Marketing Research, 26: 351.

Rosenthal, J.A., X. Lu, P. Cram (2013), 'Availability of Consumer Prices From US Hospitals for a Common Surgical Procedure', JAMA Internal Medicine, 173: 427.

Scherr, K.A., A. Fagerlin, T. Hofer, L.D. Scherer, M. Holmes-Rovner, L.D. Williamson, V.C. Kahn, J. S. Montgomery, K.L. Greene, B. Zhang, P.A. Ubel (2017), 'Physician Recommendations Trump Patient Preferences in Prostate Cancer Treatment Decisions', Medical Decision Making, 37: 56-69.

Shiv, B., Z. Carmon, D. Ariely (2005), 'Placebo Effects of Marketing Actions: Consumers May Get What They Pay For', Journal of Marketing Research, 42: 383-393.

Sinaiko, A.D., M.B. Rosenthal (2011), 'Increased Price Transparency in Health Care: Challenges and Potential Effects', New England Journal of Medicine, 364:891-894.

Slovic, P. (1995), 'The construction of preference', American Psychologist, 50: 364-371.

Ubel, P.A., C.J. Zhang, A. Hesson, J.K. Davis, C. Kirby, J. Barnett, W.G. Hunter (2016), 'Study Of Physician And Patient Communication Identifies Missed Opportunities To Help Reduce Patients Out-Of-Pocket Spending', Health Affairs, 35: 654-661.

Waber, R.L., B. Shiv, Z. Carmon, D. Ariely (2008), 'Commercial Features of Placebo and Therapeutic Efficacy', JAMA Internal Medicine, 299: 1016-1017. 